This is the author's copy of the publication as archived with the DLR's electronic library at http://elib.dlr.de . Please consult the original publication for citation.

\title{
Lyapunov-based Control Allocation for Over-actuated Nonlinear Systems
}

\section{R. de Castro and J. Brembeck}

This paper deals with the distribution of control effort in over-actuated nonlinear systems using control allocation (CA) methods. The developed CA methods rely on constrained optimization and have two key features. First, Lyapunov-based constraints and cost function are inserted into the optimization problem to improve the controller's response to unattainable virtual inputs. Second, the CA formulation is enhanced with control barrier functions to enforce state constraints. The effectiveness of the proposed CA methods is demonstrated through simulation tests of a motion controller for an over-actuated road vehicle.

\section{Copyright Notice}

C2020 IEEE. Personal use of this material is permitted. Permission from IEEE must be obtained for all other uses, in any current or future media, including reprinting/republishing this material for advertising or promotional purposes, creating new collective works, for resale or redistribution to servers or lists, or reuse of any copyrighted component of this work in other works.

R. de Castro and R. Brembeck, " Lyapunov-based Control Allocation for Over-actuated Nonlinear Systems," 2019 American Control Conference (ACC), 2019, 10.23919/ACC.2019.8814326. 


\title{
Lyapunov-based Control Allocation for Over-actuated Nonlinear Systems
}

\author{
Ricardo de Castro and Jonathan Brembeck
}

\begin{abstract}
This paper deals with the distribution of control effort in over-actuated nonlinear systems using control allocation (CA) methods. The developed CA methods rely on constrained optimization and have two key features. First, Lyapunov-based constraints and cost function are inserted into the optimization problem to improve the controller's response to unattainable virtual inputs. Second, the CA formulation is enhanced with control barrier functions to enforce state constraints. The effectiveness of the proposed CA methods is demonstrated through simulation tests of a motion controller for an over-actuated road vehicle.
\end{abstract}

\section{INTRODUCTION}

Over-actuation is a common characteristic in the motion control of many mechanical systems, such as aircrafts [1], road vehicles [2] and ships [3]. The increase in the number of actuators enhances fault-tolerant capability, control accuracy, and dynamic response of these systems [1], but also complicates the control design task. To simplify this design task, a modular control structure composed of two levels is usually adopted. The first level, also known as the highlevel controller, manipulates a reduced number of virtual inputs - such as the center-of-gravity accelerations of a road vehicle - in order to fulfill primary control objectives. In the second level, a control allocation (CA) module distributes the control effort among the actuators (e.g. braking torques and steering angles in a road vehicle) such that the virtual input is executed by the system.

Taking into account the multitude of constraints and objectives of the CA, numerical optimization approaches in the form of linear programming, quadratic programming and non-linear optimization - are commonly found in the literature [1]. Another approach to tackle the CA problem, pioneered by Johansen [4], consists in employing control Lyapunov-functions (CLF) to derive dynamic update laws for the CA problem. The key advantage of this approach is the numerical efficiency, since no iterative solver is needed to cope with the CA optimization problem. Spurred by this seminal work, further extensions were proposed to handle internal dynamics [5], parameter uncertainty [6], and output feedback problems [7]. These CLF-based approaches are revisited here from a different perspective. In that sense, our goal is to preserve the numerical optimization formulation in the CA, but augment it with Lyapunov-based constraints and costs. This augmentation, named Lyapunov-based CA (LCA), provides the following two important contributions.

The authors are with Institute of System Dynamics and Control, German Aerospace Center (DLR), Münchner Strasse 20, 82234 Wessling, Germany Ricardo.DeCastro@dlr.de
The first contribution is related with the handling of unfeasible (or unattainable) virtual inputs in the CA, which might appear due to actuator saturation or faults. As discussed in the survey [1], the majority of the optimization-based CA formulations handle these unfeasible cases by degrading the control performance. This degradation might negatively affect the transient response and stability of the overall control system. The LCA formulation proposed in this work mitigates these risks by augmenting the allocation problem with an additional Lyapunov-based cost term that penalizes the ultimate bound of the controller's output.

The second contribution of this work deals with the system's safety. More specifically, control barrier functions (CBF) [8] are incorporated into the CA formulation in order to enforce state-base constraints. In the context of over-actuated systems, the combination of CA with CBF is particularly useful, because it allows us to exploit the null space of the control input to enforce safety constraints, while having a minimal impact upon the controller performance.

\section{PRELIMINARIES}

\section{A. Notation}

The Euclidean norm of the vector $x \in \mathbb{R}^{n_{x}}$ is represented as $\|x\|$. The derivative of the scalar function $h(x)$ along the vector field $f(x)$ will be expressed using Lie derivatives, i.e., $\dot{h}=L_{f} h(x) \triangleq \frac{\partial h(x)}{\partial x} f(x)$.

\section{B. Problem Formulation}

Consider the following non-linear model [9]

$$
\begin{aligned}
\dot{x} & =f_{x}(x)+G_{x}(x) u+d \\
z & =C x-r
\end{aligned}
$$

where $x \in \mathcal{X} \subset \mathbb{R}^{n_{x}}$ is the system state, $u \in \mathcal{U} \subset \mathbb{R}^{n_{u}}$ the control input and $d \in \mathcal{D} \subset \mathbb{R}^{n_{x}}$ an additive disturbance. The sets $\mathcal{X}, \mathcal{U}, \mathcal{D}$ represent the state domain, the control input space and the set of admissible disturbances, respectively. The output $z \in \mathbb{R}^{n_{z}}$ expresses the tracking error with respect to the reference signal $r \in \mathcal{R} \subset \mathbb{R}^{n_{z}}$, which is assumed to have a bounded first-order derivative $(\dot{r})$ and lie in the reference domain $\mathcal{R}$. Furthermore, the above model assumes the functions $f_{x}(x) \in \mathbb{R}^{n_{x}}, G_{x}(x) \in \mathbb{R}^{n_{x} \times n_{u}}$ to be sufficient smooth and a constant output matrix $C \in \mathbb{R}^{n_{z} \times n_{x}}$.

The primary control goal is to find a feedback control law for $u \in \mathcal{U}$ such that the error $z$ converges to a small neighborhood around the origin for all admissible disturbances $d \in \mathcal{D}$. To achieve this goal, the output dynamics will be represented as:

$$
\dot{z}=C f_{x}(x)-\dot{r}+C G_{x}(x) u+C d
$$


We consider over-actuated systems, where the number of control inputs $\left(n_{u}\right)$ is higher than the controlled degrees of freedom $\left(n_{\tau}\right)$. As a result, it is assumed that $\operatorname{rank} C G_{x}(x)=$ $n_{\tau}<n_{u}, \forall x \in \mathcal{X}$, i.e., the matrix $C G_{x}(x)$ does not possess full column rank. This allows us to factorize the matrix $C G_{x}(x)$ as [9]:

$$
C G_{x}(x)=G_{z}(x) G(x)
$$

where $G_{z}(x) \in \mathbb{R}^{n_{z} \times n_{\tau}}$ and $G(x) \in \mathbb{R}^{n_{\tau} \times n_{u}}$ both have rank $n_{\tau}$. It also allows us write the output dynamics as:

$$
\begin{aligned}
\dot{z} & =f_{z}(x, \dot{r})+G_{z}(x) \tau+C d \\
\tau & =G(x) u
\end{aligned}
$$

where $f_{z}(x, \dot{r})=C f_{x}(x)-\dot{r}$ and $\tau \in \mathbb{R}^{n_{\tau}}$ is the virtual control input. The system's zero-dynamics [10] - composed by the states that are not directly controllable by $\tau$ - are assumed to be stable. Thanks to the introduction of the virtual control input, the control problem can be divided in two steps. In the first step, a high-level controller manipulates the virtual input $\tau$ in order to fulfill the primary control goal. In the second step, referred hereafter as the $C A$ problem, the computed virtual input $\tau$ is mapped into the control input space $\mathcal{U}$.

Since the $C A$ problem is the main focus of this work, in what follows, we will assume the existence of a baseline control law $\left(\tau_{b}(z)\right)$ for the high-level controller, as well as an accompanying Lyapunov function, $V_{b}(z)$, satisfying

$$
\begin{aligned}
\dot{V}_{b}(z, d) & =L_{f_{z}} V_{b}(z)+L_{G_{z}} V_{b}(z) \tau_{b}(z)+L_{C} V_{b}(z) d \\
& \leq-c_{V} V_{b}(z), \quad \forall\|z\| \geq \mu_{z}, d \in \mathcal{D}
\end{aligned}
$$

$\forall z \in \mathcal{Z}$, with the output domain $\mathcal{Z}=\left\{z \in \mathbb{R}^{n_{z}}, z=C x-r, x \in\right.$ $\mathcal{X}\}$, and positive constants $c_{V}, \mu_{z}$. This assumption ensures that the tracking error $z$ is ultimately bounded (see [10]). The design of the high-level control law $\tau_{b}(z)$ can be based on different types of nonlinear control methods - such as inputoutput linearization, sliding mode control, backstepping while converse Lyapunov theorems provide conditions for the existence of the function $V_{b}(z)$. The interested reader is referred to [10] for details.

The solution space of the CA problem is characterized by a set of underdetermined equations:

$$
\mathcal{U}_{C A}(\tau, x)=\{u \in \mathcal{U}: \tau=G(x) u\}
$$

If the virtual input is feasible $\left(\mathcal{U}_{C A}\left(\tau_{b}, x\right) \neq \emptyset\right)$, multiple solutions for the $C A$ problem normally exist. This multitude of solutions can be combined with optimization-based strategies to pursue secondary control goals, e.g. minimization of power consumption, actuator wear, or other criteria [1]. On the other hand, there might be situations where the requested virtual input is unfeasible $\left(\mathcal{U}_{C A}\left(\tau_{b}, x\right)=\emptyset\right)$, e.g. due to actuation saturations or faults. In these cases, the CA injects a correction term $\left(\Delta \tau \in \mathbb{R}^{n_{\tau}}\right)$ such that the resulting virtual input $\tau=\tau_{b}+\Delta \tau$ is feasible (i.e., $\mathcal{U}_{C A}\left(\tau_{b}+\Delta \tau, x\right) \neq \emptyset$ ) and the magnitude of the correction term minimized. Based on these considerations, the classical CA (CCA) problem can be formulated as [1]:

$\min _{u, \Delta \tau} J_{\tau}(\Delta \tau)+J_{u}(u), \quad$ s.t. $\quad \tau_{b}+\Delta \tau=G(x) u, \quad u \in \mathcal{U}$ where the cost $J_{\tau}(\Delta \tau)=\Delta \tau^{T} W_{\Delta} \Delta \tau$ penalizes allocation errors, $J_{u}(u)$ penalizes secondary actuation criteria and $W_{\Delta}$ is a positive definite weight matrix, which allows the designer to assign different penalization factors to each channel in the virtual input.

\section{LyAPUNOV-BASED CONTROL AlLOCATION (LCA)}

\section{A. Motivation}

The main goal of the Lyapunov-based CA (LCA) is to improve the CCA's response when unfeasible virtual demands are requested, i.e., $\mathcal{U}_{C A}\left(\tau_{b}, x\right)=\emptyset$. As previously explained, these unfeasible situations force the CCA to inject an allocation error $\Delta \tau$, deviating the virtual input from the desired baseline value. This deviation, besides degrading the controller's performance, might also negatively affect the stability of the overall control system. To better understand this issue, the allocation error $\Delta \tau$ will be treated as a perturbation and its effect in the overall stability of the closed-loop system will be analyzed using Lyapunov methods.

Consider $V=V_{b}(z)$ as candidate Lyapunov function for the closed-loop system with $\tau=\tau_{b}+\Delta \tau$. Condition (7a) can be re-written as:

$$
\begin{aligned}
\dot{V} & =L_{f_{z}} V(z)+L_{G_{z}} V(z) \tau_{b}(z)+L_{C} V(z) d+L_{G_{z}} V(z) \Delta \tau \\
& \leq-c_{V} V(z)+\Delta_{\dot{V}}, \quad \forall\|z\| \geq \mu_{z}, d \in \mathcal{D}
\end{aligned}
$$

where $\Delta_{\dot{V}}=L_{G_{z}} V(z) \Delta \tau$ represents the perturbation caused by the allocation error. Depending on the sign of $\Delta_{\dot{V}}$, this perturbation might have a "good" or "bad" effect on the performance of the controller. For example, a "good" perturbation is obtained when $\Delta_{\dot{V}}<0$; in this case, $\Delta_{\dot{V}}$ makes $\dot{V}$ even more negative, which might improve the transient response of the controller. On the other hand, "bad" perturbations are generated when $\Delta_{\dot{V}}>0$. In this case, the output's ultimate bound might increase and, in the worstcase scenario, compromise the closed-loop stability of the system.

\section{B. LCA Formulation}

In light of the previous observation, it is important to limit positive values of $\Delta_{\dot{V}}$. Accordingly, let us upper bound the term $\Delta_{\dot{V}}$ with a non-negative constant $s$, i.e.,

$$
\Delta_{\dot{V}}=L_{G_{z}} V(z) \Delta \tau \leq s, \quad s \geq 0
$$

The LCA attempts to attenuate the effects of "bad" perturbations by: $i$ ) incorporating (11) as an additional constraint into the CA's problem; and ii) augmenting the CA's cost function with the term $s^{2}$ to actively penalize (non-negative) maximum values of the perturbation $\Delta_{\dot{V}}$. As a result, the LCA is formulated as:

$$
\begin{gathered}
\left(u^{*}, \Delta \tau^{*}, s^{*}\right)=\underset{u, \Delta \tau, s}{\operatorname{argmin}} \quad J_{s}(s)+J_{\tau}(\Delta \tau)+J_{u}(u) \\
\text { s.t. } \quad \tau_{b}(z)+\Delta \tau=G(x) u, \quad u \in \mathcal{U} \\
L_{G_{z}} V(z) \Delta \tau \leq s, \quad s \geq 0
\end{gathered}
$$

where $J_{s}(s)=w_{s} s^{2}, w_{s}>0$ is a weight parameter and $\left(u^{*}, \Delta \tau^{*}, s^{*}\right)$ the optimal solution. 


\section{SAFE Lyapunov-BASEd CONTROL Allocation}

In this section the LCA will be enhanced with an additional feature: fulfillment of state-base constraints. These constraints are formulated through a safe set $\mathcal{C} \subset \mathcal{X}$, defined as the superlevel set of the scalar function $h(x)$ :

$$
\mathcal{C}=\{x \in \mathcal{X}: h(x) \geq 0\}
$$

From a technical point of view, forward invariance of the safe set $\mathcal{C}$ is desired, i.e., if $x(0) \in \mathcal{C}$ then $x(t) \in \mathcal{C}, \forall t \geq 0$.

\section{A. Barrier Functions}

To ensure forward invariance of $\mathcal{C}$, barrier functions are employed. In the literature, two classes of barrier functions are normally used [8]: $i$ ) barrier functions that are unbounded on the boundary of $\mathcal{C}$ and $i$ ) barriers functions that vanish on the boundary of $\mathcal{C}$. Given that unbounded barrier values are inconvenient for real-time implementations, the latter class - in the form of zeroing control barrier function (ZCBF) will be employed here.

Definition 1. [11] Consider the set $\mathcal{C} \subset \mathcal{X} \subset \mathbb{R}^{n_{x}}$ defined by (13) for the function $h(x): \mathcal{X} \rightarrow \mathbb{R}$. The function $h(x)$ is a zeroing control barrier function $(Z C B F)$ if there is an extended class $K$ function ${ }^{1} \alpha_{b}$ such that

$$
\sup _{u \in \mathcal{U}} \inf _{d \in \mathcal{D}}\left[L_{f_{x}} h(x)+L_{G_{x}} h(x) u+L_{I} h(x) d+\alpha_{b}(h(x))\right] \geq 0,
$$

$\forall x \in \mathcal{X}$, where $L_{I} h_{j}(x)$ is the Lie derivative over the identity matrix $I \in \mathbb{R}^{n_{x} \times n_{x}}$.

It is assumed that $L_{G_{x}} h(x) \neq 0$, i.e., the relative degree of $h$ w.r.t. $u$ is 1 . The construction of barrier functions for higher relative degrees is technically possible, but is more involved (see [8] for details). We will also assume that a lower bound - possibly state dependent - for the disturbance $d$ can be computed. This lower bound, called in the sequel $\underline{\varepsilon}_{d}(x)$, is defined as $\inf _{d \in \mathcal{D}} L_{I} h(x) d \geq \underline{\varepsilon}_{d}(x)$. Based on this bound, a (conservative) estimate for the admissible set of control inputs associated with the ZCBF can be determined as:

$$
\begin{array}{r}
\mathcal{U}_{\text {ZCBF }}(x)=\left\{u \in \mathcal{U}: L_{f_{x}} h(x)+L_{G_{x}} h(x) u+\right. \\
\left.\underline{\varepsilon}_{d}(x)+\alpha_{b}(h(x)) \geq 0\right\}
\end{array}
$$

Lemma 1. [11] Consider the safe set $\mathcal{C}$ defined by (13) and the ZCBF $h$. Any Lipschitz continuous controller u such that $u \in \mathcal{U}_{Z C B F}(x) \neq \emptyset$ for the system (1) renders the set $\mathcal{C}$ forward invariant.

\section{B. SLCA Formulation}

In light of the previous Lemma, forward invariance of $\mathcal{C}$ can be achieved by forcing the control input $u$ to lie in the space $\mathcal{U}_{Z C B F}(x)$. This condition can be straightforwardly fulfilled by incorporating $\mathcal{U}_{Z C B F}(x)$ as an additional input constraint in the LCA problem. The resulting optimization

\footnotetext{
${ }^{1} \mathrm{~A}$ continuous function $\alpha_{b}:(-b, a) \longrightarrow \mathbb{R}$ for some $a, b>0$ is an extended class $K$ function if $i) \alpha_{b}(0)=0$; and $\left.i i\right) \alpha_{b}$ is strictly increasing[11]
}

problem, named safe Lyapunov-based CA (SLCA) hereafter, is formulated as:

$$
\begin{array}{ll}
\min _{u, \Delta \tau, s} & J_{s}(s)+J_{\tau}(\Delta \tau)+J_{u}(u) \\
\text { s.t. } & \tau_{b}+\Delta \tau=G(x) u, \quad u \in \mathcal{U} \\
& L_{G_{z}} V(z) \Delta \tau \leq s, \quad s \geq 0 \\
& L_{f_{x}} h(x)+L_{G_{x}} h(x) u+\underline{\varepsilon}_{d}(x)+\alpha_{b}(h(x)) \geq 0
\end{array}
$$

This formulation enable us to exploit the system's overactuation, in particular its null space $\mathcal{U}_{C A}(0, x)$, to simultaneously pursue multiple objectives: $i$ ) forward invariance of the safe set $\mathcal{C}$ (via $\mathcal{U}_{Z C B F}$ ), ii) minimization of the output's ultimate bound (via $J_{S}$ ), iii) minimization of the allocation error (via $J_{\tau}$ ) and actuation cost (via $J_{u}$ ). It also allows us to address conflicts between different objectives. For example, there might be occasions when the fulfillment of the state constraints leads to a violation of the baseline virtual input (i.e., $\mathcal{U}_{Z C B F}(x) \cap \mathcal{U}_{C A}\left(\tau_{b}, x\right)=\emptyset$ ). The SLCA resolves these conflicts by providing arbitration features through numerical optimization.

\section{Relaxed SLCA}

Despite the permissible nature of the ZCBF (i.e., the barrier function remains bounded even when $x$ approaches the boundary of $\mathcal{C}$ ), in practice there might be situations where the input constraint (14) might be too restrictive and produce unfeasible optimization problems $\left(\mathcal{U}_{\text {ZCBF }}(x)=\right.$ $\emptyset)$. Such situations might occur, for example, due to nonmodelled dynamics, measurements noise and other disturbances. To recover the CA's feasibility, the safe set $\mathcal{C}$ will be treated as a "soft-constraint" and $\mathcal{U}_{Z C B F}$ relaxed through the introduction of a slack variable (referred here as dilation factor). To formalize these ideas, let us consider the dilated safe set $\tilde{\mathcal{C}}$ and dilated input constraint $\tilde{\mathcal{U}}_{\text {ZCBF }}$

$$
\begin{aligned}
& \tilde{\mathcal{C}}\left(h_{d}\right)=\left\{x \in \mathcal{X} \subset \mathbb{R}^{n_{x}}: \quad h(x) \geq-h_{d}\right\} \\
& \tilde{\mathcal{U}}_{Z C B F}\left(x, s_{b}\right)=\left\{u \in \mathcal{U}: L_{f_{x}} h(x)+L_{G_{x}} h(x) u+\right. \\
& \left.\underline{\varepsilon}_{d}(x)+\alpha_{b}(h(x)) \geq-s_{b}\right\}
\end{aligned}
$$

where $h_{d}, s_{b} \geq 0$ are non-negative dilation factors. These sets fulfill $\mathcal{C} \subset \tilde{\mathcal{C}}\left(h_{d}\right), \mathcal{U}_{Z C B F}(x) \subset \tilde{\mathcal{U}}_{Z C B F}\left(x, s_{b}\right), \forall x \in \mathcal{X}$. The relaxed SLCA is formulated by replacing $\mathcal{U}_{Z C B F}(x)$ with its relaxed counterpart $\tilde{\mathcal{U}}_{Z C B F}\left(x, s_{b}\right)$, while quadratically penalizing the dilation factor $s_{b}$. This yields

$$
\begin{array}{ll}
\left(u^{*}, \Delta \tau^{*}, s^{*}, s_{b}^{*}\right)=\underset{u, \Delta \tau, s, s_{b}}{\operatorname{argmin}} J_{s_{b}}\left(s_{b}\right)+J_{s}(s)+J_{\tau}(\Delta \tau)+J_{u}(u) \\
\text { s.t. } \quad \tau_{b}(z)+\Delta \tau=G(x) u, \quad u \in \mathcal{U} \\
\quad L_{G_{z}} V(z) \Delta \tau \leq s, \quad s \geq 0 \\
\quad L_{f_{x}} h(x)+L_{G_{x}} h(x) u+\underline{\varepsilon}_{d}(x)+\alpha_{b}(h(x)) \geq-s_{b} \\
\quad s_{b} \geq 0
\end{array}
$$

where $J_{s_{b}}\left(s_{b}\right)=w_{b} s_{b}^{2}$ and $w_{b}$ is a weight that penalizes the dilation factor $s_{b}$. This dilation factor affects the size of the (dilated) safe set $\tilde{\mathcal{C}}\left(h_{d}\right)$ to where the state is attracted. The safe CA formulations above discussed consider only a single safe set $\mathcal{C}$. If multiple safe sets exist, i.e., $\mathcal{C}=\cap_{j=1}^{n_{C}} \mathcal{C}_{j}$, 
then the allowed set of control inputs, $\mathcal{U}_{Z C B F}=\cap_{j=1}^{n_{C}} \mathcal{U}_{Z C B F, j}$ (or their relaxed counterparts) must be incorporated into the allocation problem as additional input constraints. Further, in the relaxed SCLA case, multiple dilation factors $s_{b, i}$ are needed (one per safe set), which leads to the extend cost function $J_{s_{b}}=w_{b} \sum_{i=j}^{n_{C}} s_{b, j}^{2}$.

\section{Application Example}

\section{A. Modelling and Problem Formulation}

To validate the Lyapunov-based CA formulations, the motion control of an over-actuated road vehicle will be considered in this section. The dynamic model of the vehicle is represented as [2]

$$
\begin{aligned}
M \dot{x} & =M f_{v}(x)+B F_{x y}+M d \\
f_{v}(x) & =\left[\begin{array}{lllllllll}
v_{y} v_{\psi} & -v_{x} v_{\psi} & 0
\end{array}\right]^{T} \\
B & =\left[\begin{array}{cccccccccc}
1 & 0 & 1 & 0 & 1 & 0 & 1 & 0 \\
0 & 1 & 0 & 1 & 0 & 1 & 0 & 1 \\
-\frac{c}{2} & l_{f} & \frac{c}{2} & l_{f} & -\frac{c}{2} & -l_{r} & \frac{c}{2} & -l_{r}
\end{array}\right] \\
F_{x y} & =\left[\begin{array}{lllllllll}
F_{x 1} & F_{y 1} & F_{x 2} & F_{y 2} & F_{x 3} & F_{y 3} & F_{x 4} & F_{y 4}
\end{array}\right]
\end{aligned}
$$

where $x=\left[\begin{array}{lll}v_{x} & v_{y} & v_{\psi}\end{array}\right]^{T}$ is the vehicle's velocity vector, containing the longitudinal $\left(v_{x}\right)$, lateral $\left(v_{y}\right)$, and yaw $\left(v_{\psi}\right)$ velocities, while $F_{x i}$ and $F_{y i}$ represent the longitudinal and lateral forces generated by the tire $i \in\{1,2,3,4\}^{2}$, respectively. The matrices $M=\operatorname{diag}\left(m, m, I_{z}\right)$ and $B$ are dependent on the vehicle's inertial and geometric parameters $m, I_{z}, l_{f}, l_{r}, c$, which are defined in Appendix. Modeling approximation errors and perturbations are embedded in the bounded additive disturbance $d \in \mathcal{D}=\left\{d \in \mathbb{R}^{3}:\|d\| \leq \bar{d}\right\}$, where $\bar{d}$ is a known upper bound.

The vehicle's front axle is assumed to have fully actuated wheels, i.e., with independent steering, braking and traction actuators, while the rear wheels are equipped with individual braking actuators. Due to this highly-actuated structure, the longitudinal and lateral forces of the front tires $\left(F_{x i}, F_{y i}, i=\right.$ $1,2)$ and the rear longitudinal forces $\left(F_{x i}, i=3,4\right)$ are assumed to be directly controlled. These forces are constrained by friction ellipse constraints [12] and physical actuation limits:

$$
\begin{aligned}
& u=\left[\begin{array}{llllll}
F_{x 1} & F_{y 1} & F_{x 2} & F_{y 2} & F_{x 3} & F_{x 4}
\end{array}\right] \in \mathcal{U} \\
& \mathcal{U}=\left\{u \in \mathbb{R}^{6}: \quad\left(\frac{F_{x i}}{d_{x i}}\right)^{2}+\left(\frac{F_{y i}}{d_{y i}}\right)^{2} \leq\left(\mu_{i} F_{z i}\right)^{2}, i=1,2\right. \\
& \underline{F_{x i}} \leq F_{x i} \leq \overline{F_{x i}}, \quad i=1,2 \\
& \left.\underline{F_{x i}} \leq F_{x i} \leq 0, \quad i=3,4\right\}
\end{aligned}
$$

where $\mu_{i}$ is the tire-road friction peak, $F_{z i}$ the vertical load of the tire, $d_{x i}$ and $d_{y i}$ constants, and $\left[\underline{F_{x i}}, \overline{F_{x i}}\right]$ force limits. As explained in [2], [13], model inversion methods can be used to map the desired forces $u$ into actuator demands.

To model the coupling between the lateral and longitudinal forces of the rear tires, the magic tire formula [12] and the

\footnotetext{
${ }^{2}$ index 1 refers to the front left tire, 2 to the front right, 3 to the rear left, and 4 to the rear right tire
}

friction ellipse are employed:

$$
\begin{aligned}
F_{y i}\left(x, F_{x i}\right) & =\tilde{F}_{y i}(x) \varphi\left(F_{x i}\right), \varphi\left(F_{x i}\right)=\sqrt{1-\left(\frac{F_{x i}}{\mu_{i} F_{z i}}\right)^{2}} \\
\tilde{F}_{y i}(x) & =\mu_{i} F_{z i} \sin \left(C_{i} \operatorname{atan}\left(B_{i} \alpha_{i}(x)\right)\right) \\
\tan \left(\alpha_{i}(x)\right) & =-\frac{\vartheta_{y i}^{T} x}{\vartheta_{x i}^{T} x}, \quad\left[\begin{array}{l}
\vartheta_{x i}^{T} \\
\vartheta_{y i}^{T}
\end{array}\right]=\left[\begin{array}{lll}
1 & 0 & \chi_{x i} \\
0 & 1 & \chi_{y i}
\end{array}\right] \\
{\left[\begin{array}{ll}
\chi_{x 3} & \chi_{x 4}
\end{array}\right] } & =\left[\begin{array}{ll}
-\frac{c}{2} & \frac{c}{2}
\end{array}\right], \quad\left[\begin{array}{lll}
\chi_{y 3} & \chi_{y 4}
\end{array}\right]=\left[\begin{array}{ll}
-l_{r} & -l_{r}
\end{array}\right]
\end{aligned}
$$

for $i=3,4$, where $B_{i}, D_{i}$ are parameters and $\alpha_{i}(x)$ the side-slip angle of the tire $i$. The term $\tilde{F}_{y i}$ represents the lateral force generated by the rear tire $i$ under pure sideslip conditions. It is "derated" by the factor $\varphi\left(F_{x i}\right) \in[0,1]$ when longitudinal forces are applied.

The primary control goal of the vehicle's motion controller is to find reference forces $u \in \mathcal{U}$ such that the velocities $\left[\begin{array}{ll}v_{x} & v_{\psi}\end{array}\right]^{T}$ follow the reference signal $r=\left[\begin{array}{cc}v_{x}^{*} & v_{\psi}^{*}\end{array}\right]^{T}$ as close as possible. To exploit the vehicle's over-actuation, the motion controller should minimize the tires' friction usage [2]:

$J_{u}(u)=\sum_{i \in\{1,2\}} \frac{F_{x i}^{2}+F_{y i}^{2}}{\left(\mu_{i} F_{z i}\right)^{2}}+\sum_{i \in\{3,4\}} \frac{F_{x i}^{2}+\left(\tilde{F}_{y i}(x)^{2}\left(1-\frac{F_{x i}}{\mu_{i} F_{z i}}\right)\right)}{\left(\mu_{i} F_{z i}\right)^{2}}$

This secondary control goal penalizes the controlled forces with a factor inversely proportional to the tire's vertical load, promoting a balanced use of the frictional potential in the tires. To further enhance the vehicle's stability, the motion controller should also protect the side-slip angles of the rear tires [13]; accordingly, the following limits must be enforced:

$C=\left\{x \in \mathbb{R}^{3}: \tan (-\bar{\alpha}) \leq \tan \left(\alpha_{i}(x)\right) \leq \tan (\bar{\alpha}), \quad i=3,4\right\}$

where $\bar{\alpha}$ is maximum allowed side-slip angle. After some algebraic manipulations, these constraints can be represented as an intersection of polytopic sets, $C=\cap_{j=1}^{n_{c}} C_{j}$,

$$
C_{j}=\left\{x: h_{j}(x)=q_{j}^{T} x \geq 0\right\}, \quad j=1, \ldots, n_{c}
$$

with the vector $q_{j} \in \mathbb{R}^{3}$ defined in Table I.

To facilitate the handling of the force coupling between longitudinal and lateral rear forces, the derating factor $\varphi\left(F_{x i}\right)$ will be approximated by $\varphi\left(F_{x i}\right) \approx 1+\frac{F_{x i}}{\mu_{i} F_{z i}}$, which is valid in the domain $F_{x i} \in\left[F_{x i}, 0\right], i=3,4$. This pragmatic approximation allows us to obtain an affine mapping between $F_{x y}$ and the control input $u$ :

$$
F_{x y} \approx B_{u}(x) u+f_{y}(x)
$$

where $B_{u}(x)$ and $f_{y}(x)$ are defined in [14]. Based on these considerations, the vehicle dynamics model can now be compactly described as:

$$
\begin{aligned}
\dot{x} & =\underbrace{f_{v}(x)+M^{-1}\left(B f_{y}(x)\right)}_{f_{x}(x)}+\underbrace{M^{-1} B B_{u}(x)}_{G_{x}(x)} u+d \\
z & =\left[\begin{array}{lll}
1 & 0 & 0 \\
0 & 0 & 1
\end{array}\right] x-r=C x-r \\
\tau & =G_{z}^{-1} C G_{x}(x) u=G(x) u, \quad G_{z}=\left[\begin{array}{ll}
1 & 0 \\
0 & 1
\end{array}\right]
\end{aligned}
$$


TABLE I

SUMMARY OF THE LCA/SLCA FORMULATIONS

\begin{tabular}{|ll|l|}
\hline$L_{G_{z}} V(z)$ & $=2 z^{T} P G_{z}$ \\
$h_{j}(x)$ & $=$ & $q_{j}^{T} x$ \\
$L_{f_{x}} h_{j}(x)$ & $=$ & $q_{j}^{T} f_{x}(x)$ \\
$L_{G_{x}} h_{j}(x)$ & $=q_{j}^{T} G_{x}(x)$ \\
$\alpha_{b}\left(h_{j}(x)\right)$ & $=k_{j}\left(h_{j}(x)\right)^{3}$ \\
$\underline{\varepsilon}_{j, d}(x)$ & $=$ & $-\left\|q_{j}\right\| \bar{d}$
\end{tabular}$\quad\left[\begin{array}{l}q_{1}^{T} \\
q_{2}^{T} \\
q_{3}^{T} \\
q_{4}^{T}\end{array}\right]=\left[\begin{array}{c}\vartheta_{y 3}^{T}+\vartheta_{x 3}^{T} \tan (\bar{\alpha}) \\
-\vartheta_{y 3}^{T}-\vartheta_{x 3}^{T} \tan (-\bar{\alpha}) \\
\vartheta_{y 4}^{T}+\vartheta_{x 4}^{T} \tan (\bar{\alpha}) \\
-\vartheta_{y 4}^{T}-\vartheta_{x 4}^{T} \tan (-\bar{\alpha})\end{array}\right]$
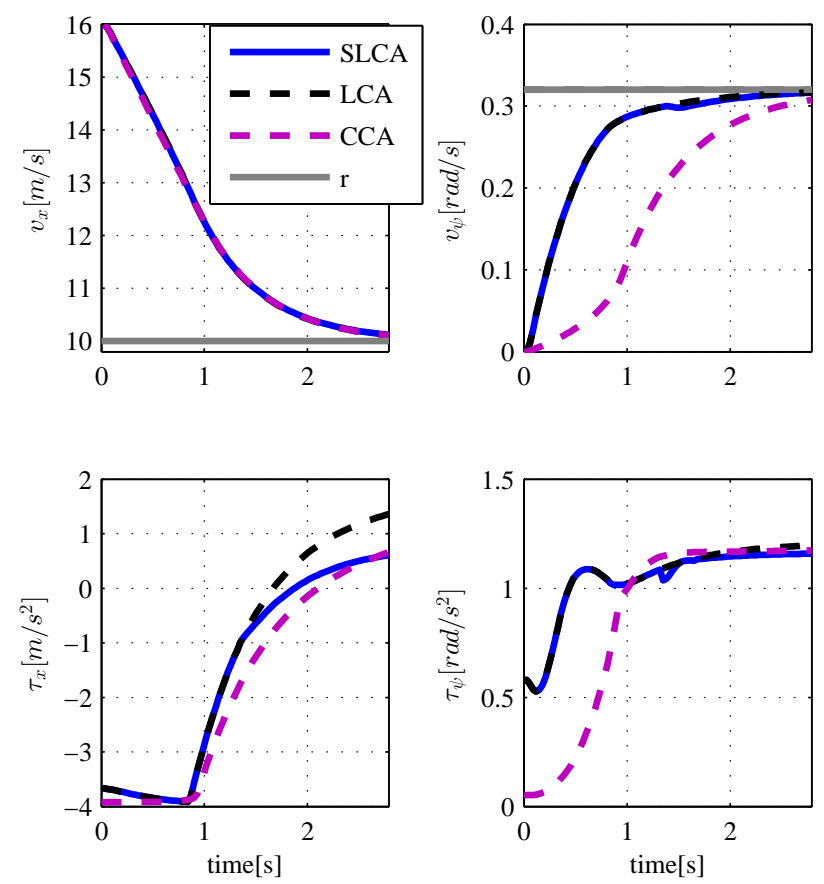

Fig. 1. Simulation results: controlled velocities and virtual inputs.

where $\tau=\left[\begin{array}{ll}\tau_{x} & \tau_{\psi}\end{array}\right]^{T}$ contains the longitudinal $\left(\tau_{x}\right)$ and yaw $\left(\tau_{\psi}\right)$ accelerations generated by the actuators.

The high-level controller relies on input-output linearization [10]. Since the relative degree ${ }^{3}$ of the output $z$ is $\left[\begin{array}{ll}1 & 1\end{array}\right]^{T}$, the following control law can be used to cancel the dominant nonlinearities in the system:

$$
\tau_{b}=G_{z}^{-1}\left(-C f_{x}(x)+\dot{r}+K z\right)
$$

where $K$ is a Hurwitz matrix selected by the designer. Taking into account the resulting linearized dynamics $(\dot{z}=K z+C d)$, a quadratic Lyapunov $V=z^{T} P z$ will be considered for the closed-loop system, where $P$ is a positive definite matrix obtained by solving the equation $P K+K^{T} P=-Q$ (with $Q$ a positive definite matrix defined by the designer). It can be easily verified that this Lyapunov function fulfills assumption (7).

\section{B. Simulation Results}

After presenting the vehicle's model and high-level controller, this final section will focus on the validation and

${ }^{3}$ recall that $z$ has a dimensionally of 2 , consequently the relative degree has to be computed for all elements in $z$
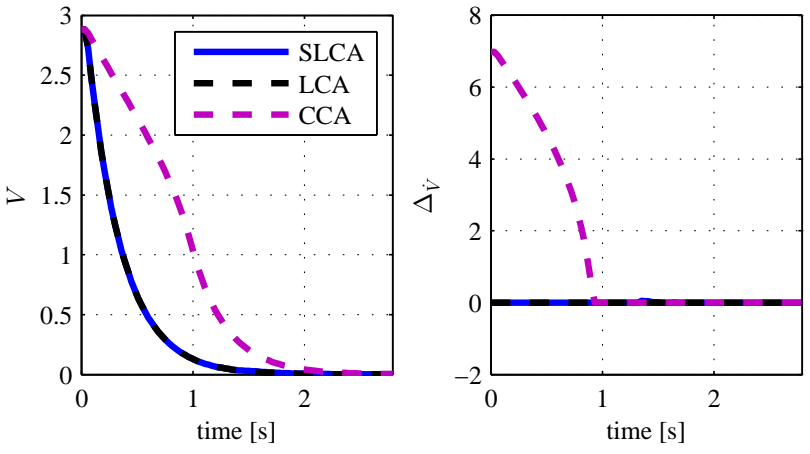

(a) Lyapunov function $(V)$ and perturbation $\Delta_{\dot{V}}$
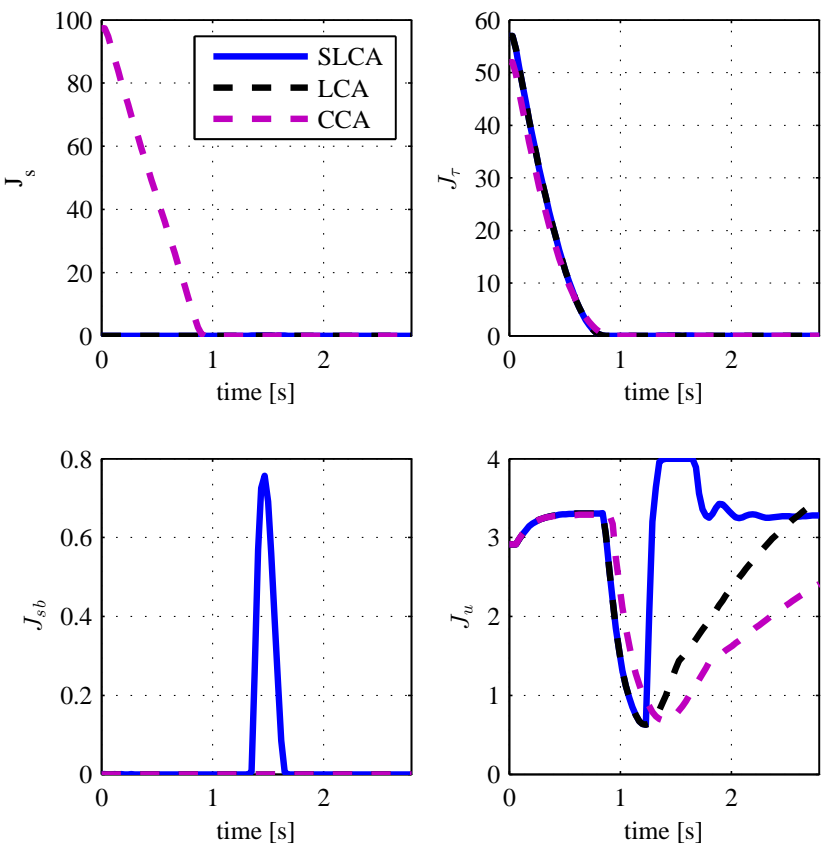

(b) cost functions of the CA problems
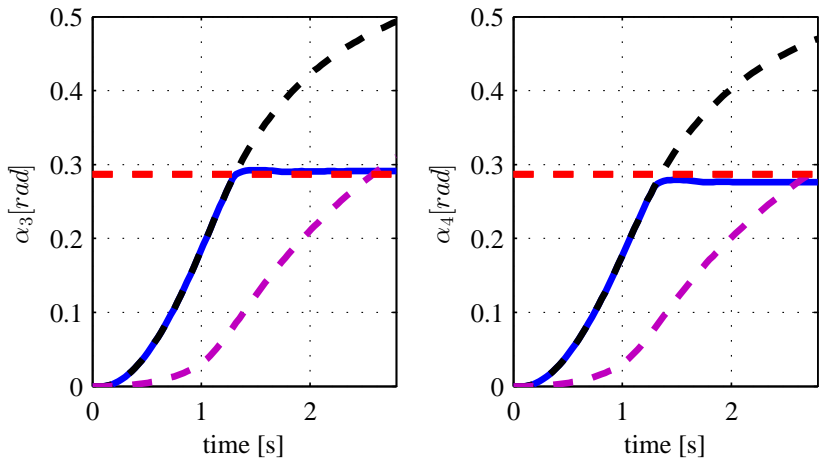

(c) side-slip angle of rear tires (red line limit the allowed boundary)

Fig. 2. Simulation results.

comparison of the CCA, LCA and relaxed SLCA strategies. To efficiently solve all the allocation problems the embedded conic solver (ECOS) [15] was employed. A summary of the LCA and SLCA parameterizations can be found in Table I and Appendix. Fig. 1 to 3 show the simulation results of the motion controller when following a constant reference under 

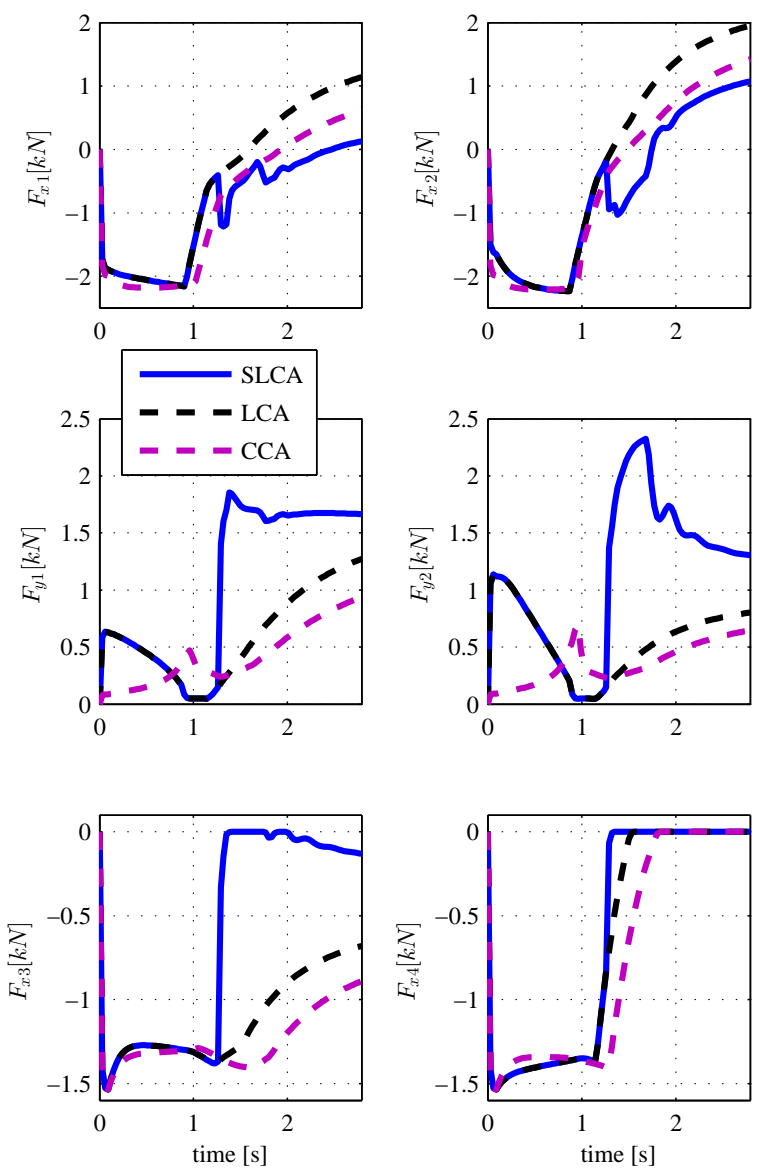

Fig. 3. Reference forces generated by the CA strategies.

low adhesion conditions $\left(\mu_{i}=0.4\right)$. To quickly decrease the velocity errors, all the motion controllers generate a combined braking and cornering accelerations that are unfeasible (note the non-zero allocation cost $J_{\tau}$ in Fig. $2 \mathrm{~b}$ ). The results also reveal that, in comparison with the CCA, the LCA and SLCA offer a faster transient response, particularly in the $v_{\psi}$ channel. For example, both the LCA and SLCA have a $v_{\psi}$ settling time (with $5 \%$ error band) of $1.56 \mathrm{~s}$, while the CCA requires $2.7 \mathrm{~s}$. The key reason for this superior transient response lies in the allocation priorities: while the CCA favors the minimization of the allocation error (see $J_{\tau}$ in Fig. 2b), the LCA and SLCA prioritizes the minimization of the disturbance $\Delta_{\dot{V}}$ via $J_{s}$. As a result, the LCA-based allocations minimizes the violation of the Lyapunov conditions (7a), which translates to a better controller's transient performance (see also the time-response of the Lyapunov function $V$, depicted in Fig. 2a).

The superior performance of the LCA comes with a price: violation of the safety constraints $\mathcal{C}$, which leads to excessive side-slip angles in the rear axle (see Fig. 2c). These dangerous operation modes can be surpassed by the SLCA. More specifically, from $1.3 \mathrm{~s}$ onwards, the SLCA limits the (rear) side-slip angles by decreasing the rear braking force and raising the force magnitude in the front axle (see Fig. 3). This means that the SLCA shifts the allocation effort from the rear to the front tires as a measure to protect the rear's side-slip angles. Interestingly, this allocation shift does not significantly affect the response of the high-level controller (i.e., both LCA and SCLA have a similar $v_{x}$ and $v_{\psi}$ behavior).

\section{CONCLUSION AND OUTLOOK}

This work proposed new approaches to the control allocation (CA) of over-actuated nonlinear systems. These approaches relied on numerical optimization combined with: i) Lyapunov-based constraints, and ii) control barrier functions. Lyapunov-based constraints allowed us to improve the controller's response to unfeasible virtual inputs, while control barrier functions enhanced the CA safety through the enforcement of state-base constraints. Finally, the Lyapunovbased (LCA) approaches were applied to the motion control of an over-actuated road vehicle. Simulation results demonstrated the superior transient response and safety properties of the LCA formulations in comparison with state-of-the-art CA algorithms. As future work, the LCA will be investigated in the context of actuator faults and control reconfiguration.

\section{APPENDIX - LCA/SLCA PARAMETERS}

The model parameters are: vehicle mass $(m=1800 \mathrm{~kg})$, yaw inertia $\left(I_{z}=4000 \mathrm{~kg} \cdot \mathrm{m}^{2}\right)$, center of gravity's position $\left(l_{f}, l_{r}\right)=(1.17,1.52) \mathrm{m}$, tire parameters $\left(B_{i}, C_{i}\right)=(35,0.9)$, friction coefficient $\left(\mu_{i}=0.4\right)$, trackwidth $(c=1.8 \mathrm{~m})$.

\section{REFERENCES}

[1] T. A. Johansen and T. I. Fossen, "Control allocation: a survey," Automatica, vol. 49, no. 5, pp. 1087-1103, 2013.

[2] R. de Castro, M. Tanelli, R. E. Araujo, and S. M. Savaresi, "MinimumTime Path-Following for Highly Redundant Electric Vehicles," IEEE Transactions on Control Systems Technology, vol. 24, no. 2, pp. 487501, Mar. 2016.

[3] T. I. Fossen and T. A. Johansen, "A Survey of Control Allocation Methods for Ships and Underwater Vehicles," in 14th Mediterranean Conference on Control and Automation, Jun. 2006, pp. 1-6.

[4] T. A. Johansen, "Optimizing nonlinear control allocation," in 43rd IEEE Conference on Decision and Control, 2004.

[5] F. Liao, K. Y. Lum, J. L. Wang, and M. Benosman, "Constrained Nonlinear Finite-Time Control Allocation," in American Control Conference, Jul. 2007, pp. 3801-3806.

[6] J. Tjonnas and T. A. Johansen, "Adaptive control allocation," Automatica, vol. 44, no. 11, pp. 2754-2765, Nov. 2008.

[7] F. Liao, K.-Y. Lum, and J. L. Wang, "Observer-based nonlinear control allocation," in American Control Conference, 2010, pp. 6077-6082.

[8] A. D. Ames, X. Xu, J. W. Grizzle, and P. Tabuada, "Control Barrier Function Based Quadratic Programs for Safety Critical Systems," IEEE Transactions on Automatic Control, 2017.

[9] O. Harkegard and S. T. Glad, "Resolving actuator redundancy: optimal control vs. control allocation," Automatica, vol. 41, no. 1, pp. 137-144, Jan. 2005.

[10] H. Khalil, Nonlinear Control, 3rd ed. Prentice Hall, 2002

[11] X. Xu, P. Tabuada, J. W. Grizzle, and A. D. Ames, "Robustness of Control Barrier Functions for Safety Critical Control," in IFAC Conference on Analysis and Design of Hybrid Systems, 2015.

[12] H. B. Pacejka, Tyre and vehicle dynamics. Butterworth-Heinemann, 2002.

[13] C. E. Beal and J. C. Gerdes, "Model Predictive Control for Vehicle Stabilization at the Limits of Handling," IEEE Transactions on Control Systems Technology, vol. 21, no. 4, pp. 1258-1269, Jul. 2013.

[14] R. de Castro and J. Brembeck, "Supervised Trajectory Tracking Control," in IEEE International Conference on Intelligent Transportation Systems (ITSC), 2018.

[15] A. Domahidi, E. Chu, and S. Boyd, "ECOS: An SOCP solver for embedded systems," in European Control Conference, 2013. 